\title{
Cysteine-rich 61-associated gene expression profile alterations in human glioma cells
}

\author{
RUI WANG ${ }^{1}, \mathrm{BO} \mathrm{WEI}^{2}, \mathrm{JUN}^{\mathrm{WEI}}{ }^{3}, \mathrm{YU} \mathrm{TIAN}^{2}$ and $\mathrm{CHAO} \mathrm{DU}^{2}$ \\ Departments of ${ }^{1}$ Radiology, ${ }^{2}$ Neurosurgery and ${ }^{3}$ Science and Education Section, \\ China-Japan Union Hospital of Jilin University, Changchun, Jilin 130033, P.R. China
}

Received January 28, 2016; Accepted February 20, 2017

DOI: $10.3892 / \mathrm{mmr} .2017 .7216$

\begin{abstract}
The present study aimed to investigate gene expression profile alterations associated with cysteine-rich 61 (CYR61) expression in human glioma cells. The GSE29384 dataset, downloaded from the Gene Expression Omnibus, includes three LN229 human glioma cell samples expressing CYR61 induced by doxycycline (Dox group), and three control samples not exposed to doxycycline (Nodox group). Differentially expressed genes (DEGs) between the Dox and Nodox groups were identified with cutoffs of llog2 fold change (FC) $>0.5$ and $\mathrm{P}<0.05$. Gene ontology and Kyoto Encyclopedia of Genes and Genomes pathway enrichment analyses for DEGs were performed. Protein-protein interaction (PPI) network and module analyses were performed to identify the most important genes. Transcription factors (TFs) were obtained by detecting the TF binding sites of DEGs using a Whole Genome rVISTA online tool. A total of 258 DEGs, including $230(89 \%)$ upregulated and $28(11 \%)$ downregulated DEGs were identified in glioma cells expressing CYR61 compared to cells without CYR61 expression. The majority of upregulated DEGs, including interferon $(I F N) B 1$, interferon-induced (IFI)44 and interferon regulatory factor $(I R F) 7$, were associated with immune, defense and virus responses, and cytokine-cytokine receptor interaction signaling pathways. Signal transducer and activator of transcription 1 (STAT1) and DEAD-box helicase 58 (DDX58) were observed to have high connection degrees in the PPI network. A total of seven TFs of the DEGs, including interferon consensus sequence-binding protein and IFN-stimulated gene factor-3 were additionally detected. In conclusion, IFNB1, genes encoding IFN-induced proteins (IFI16, IFI27, IFI44 and IFITM1), IRFs (IRF1, IRF7 and IRF9), STAT1 and DDX58 were demonstrated to be associated with CYR61 expression in glioma cells; thus, they may
\end{abstract}

Correspondence to: Dr Chao Du, Department of Neurosurgery, China-Japan Union Hospital of Jilin University, 126 Xiantai Street, Changchun, Jilin 130033, P.R. China

E-mail: duchchchaoaoao@hotmail.com

Key words: glioma cells, cysteine-rich 61, differentially expressed genes, protein-protein interaction network, module analysis be critical for maintaining the role of CYR61 during cancer progression.

\section{Introduction}

Cysteine-rich 61 (CYR61) is a secreted, cysteine-rich, heparin-binding protein (1) involved in a variety of cellular functions including adhesion, migration and proliferation (2). Previously, Xie et al (3) reported that CYR61 was overexpressed in 66 primary gliomas compared with healthy brain samples, and that CYR61 expression was significantly correlated with tumor grade and patient survival (3). CYR61-overexpressing glioma cells were observed to have an increased proliferation rate (4). Additionally, CYR61 has been revealed to be differentially expressed in numerous other tumor types $(5,6)$. Thus, it is necessary to clarify the mechanisms underlying the expression of CYR61 in human glioma cells.

A previous study suggested that CYR61 is a tumorpromoting factor and a key regulator of cancer progression (7). CYR61 appears to stimulate numerous signaling pathways in the development of gliomas. In malignant glioma cells, overexpression of CYR61 was demonstrated to enhance tumorigenicity via the integrin-linked kinase signaling pathway, including the $\beta$-catenin-T cell factor/lymphoid enhancer factor and phosphatidylinositol 3'-kinase (PI3K) signaling pathways (8). CYR61 activates protein kinase $\mathrm{B}$ (AKT) and inhibits the apoptotic effector B-cell lymphoma 2-associated death promoter protein via the PI3K signaling pathway (9). Goodwin et al (10) identified a CYR61-dependent signaling pathway [hepatocyte growth factor (HCF)-induced AKT signaling pathway], via which the activated HCF receptor mediates cell growth and migration, and prolonged signaling events in glioma cell lines. Furthermore, overexpression of CYR61-targeting microRNAs (miRs) impairs the growth (miR-136 and miR-634) and the migration (miR-155) of glioblastoma cells (11). Thus, gene expression analysis in human glioma cells may facilitate the understanding of the genes and signaling pathways associated with CYR61.

The present study used microarray data archived by Haseley et al (12) to identify differences in the gene expression profiles of between human glioma cells expressing CYR61 and those not, to understand the underlying mechanisms of CYR61 expression in human glioma cells. Differentially expressed genes (DEGs) were identified, and the interaction 
between DEG-encoded proteins was investigated, with the aim to further the understanding of CYR61-associated gene expression profile changes in human glioma cells.

\section{Materials and methods}

Affymetrix microarray data. The gene expression dataset GSE29384 was downloaded from the GeneExpression Omnibus (GEO; www.ncbi.nlm.nih.gov/geo/) database, which was annotated based on the GPL570 (HG-U133_Plus_2) Affymetrix Human Genome U133 Plus 2.0 Array platform (www. ncbi.nlm.nih.gov/geo/query/acc.cgi?acc=GPL570). Microarray data were collected from LN229 human glioma cells expressing CYR61 (via incubation with doxycycline; Dox group) and those not expressing CYR61 (no doxycycline; Nodox group), each in triplicate (12).

DEGs screening. The Bioconductor software package version 2.14 (Fred Hutchinson Cancer Research Center, Seattle, WA, USA) was used to perform the analysis of microarray data between the Dox and Nodox groups. Raw expression data were preprocessed using the robust multiarray average method (13). For each sample, the mean value of all probes mapped to the same gene was taken as the final expression value of that gene, following which 54,675 probe sets were mapped to 19,851 genes. The limma package in Bioconductor (14), a linear regression model, was applied to perform differential expression analysis between the Dox and Nodox groups. Genes with $\log 2$ fold change $(\mathrm{FC}) \mid>0.5$ and $\mathrm{P}<0.05$ were identified as DEGs.

Gene Ontology (GO) and Kyoto Encyclopedia of Genes and Genomes (KEGG) pathway enrichment analysis. Functional analysis was performed for DEGs using Database for Annotation, Visualization and Integrated Discovery (DAVID) online software (15). GO term and KEGG pathway enrichment analyses were applied to identify the primary metabolic and functional signaling pathways in DEGs involved in human gliomas expressing CYR61. False discovery rate (FDR) $<0.05$ was used for pathway enrichment analysis and other parameters were set at the default value of DAVID.

Protein-protein interaction (PPI) network construction. The online software STRING version 10.0 (www.string-db.org/)(16) was applied to analyze the interaction of proteins encoded by DEGs. All parameters were set at the default value. Finally, the PPI network was constructed using Cytoscape software version 3.2.0 (www.cytoscape.org/) (17).

Module analysis based on PPI network. Next, module analysis of PPI network was performed using ClusterONE plugin version 1.0 (National Institute of General Medical Sciences, Seattle, WA, USA), a plug-in of Cytoscape. And the genes in the modules were further subject to GO and KEGG subpathway enrichment analysis using DAVID. GO terms and KEGG subpathways with FDR $<0.05$ were selected and all other parameters were set at the default values.

Detection of transcription factors (TFs) regulating DEGs. To further understand the mechanism underlying alterations in expression levels of these DEGs, the upstream regulatory elements of up- and downregulated DEGs were detected using the online tool Whole-Genome rVISTA (18). The promoter region was set at $1,000 \mathrm{bp}$ upstream of the transcription start site. $\mathrm{P}<0.0001$ was selected as the cutoff point.

\section{Results}

DEG screening in glioma cells expressing CYR61 protein. According to the CYR61 differential expression analysis, a total of $258 \mathrm{DEGs}(\mathrm{P}<0.05$; $\mid \log 2 \mathrm{FCl}>1)$, including 230 upregulated (89\%) and 28 downregulated (11\%) DEGs, were identified. There were more upregulated than downregulated genes in human glioma cells expressing CYR61, indicating that CYR61 is more likely to mediate the upregulation of genes in glioma.

Functional enrichment analysis of up- and downregulated $D E G s$. Enrichment analyses of GO terms and KEGG subpathways were performed for both up- and downregulated DEGs. The upregulated DEGs were primarily enriched in 14 GO terms and most significantly in the response to virus $(11.5 \%$, GO: 0009615), immune response (22.5\%, GO: 0006955) and defense response (14.8\%, GO: 0006952) terms (Table I). Genes [interferon-induced (IFI) with helicase C domain 1 (IFIHI), IFI44, IFITM2, IFI35, interferon regulatory factor (IRF) 9 and IRF7] encoding interferon (IFN) and IFN-induced proteins were primarily involved in response to virus (GO: 0009615) and immune response (GO: 0006955) terms. Additionally, genes including IFNBI, IFI6 and IFI16 were enriched in the regulation of apoptosis (GO: 0042981), cell death (GO: 0010941) and proliferation (GO: 0042127) terms.

In the KEGG subpathway analysis, upregulated DEGs were primarily enriched in 4 subpathways and most significantly associated with cytokine-cytokine receptor interactions (8.1\%, hsa04060), and the cytosolic DNA-sensing (4.3\%, hsa04623), retinoic-acid inducible gene I (RIG-I) -like receptor (4.3\%, hsa04622) and Toll-like receptor signaling (4.8\%, hsa04620) pathways. IFNB1 was enriched in four subpathways and IRF7 was enriched in three subpathways (Table II). However, there were no downregulated DEGs enriched in GO terms or KEGG subpathways.

Construction of PPI network based on DEGs. To investigate interactions among glioma-associated DEGs, a PPI network containing 148 nodes and 1,656 edges was constructed. In this protein network, the top 20 proteins with greatest degrees were selected, of which signal transducer and activator of transcription 1 (STAT1) had the greatest degree (degree $=77$ ). IFN-mediated proteins, including IFIT3 (degree=66), IFIT1 (degree=63), IFI35 (degree=55), IFI44 (degree=54) and guanylate binding protein 1 (degree $=60)$ with high degrees accounted for a larger portion among the 20 proteins. Furthermore, the IFN activation factors, IRF7 (degree=67) and IRF9, (degree $=56)$ also had high degrees.

Module construction based on the PPI network. One module was constructed based on the PPI network (Fig. 1). The network module contained 78 proteins and 1,435 interactions, indicating complex associations between protein molecules. Among the proteins in this module, IFIH1, IFIT3, DEAD-box helicase 58 (DDX58), STAT1, IRF7 and IFIT1 had higher degrees. 
Table I. GO term enrichment analysis of upregulated DEGs in gene expression profiles of glioma cells expressing CYR61.

\begin{tabular}{lll}
\hline GO ID & Function & P-value \\
\hline
\end{tabular}

\begin{tabular}{|c|c|c|c|}
\hline 0009615 & Response to virus & $1.02 \mathrm{E}-22$ & $\begin{array}{l}\text { IFIH1, IL6, BST2, CYP1A1, TLR3, RSAD2, SAMHD1, IFI44, IFI16, } \\
\text { CCL5, STAT1, TRIM22, IFI35, ISG20, STAT2, DDX58, IRF9, } \\
\text { PLSCR1, ISG15, IFNB1, IRF7, EIF2AK2, MX1, MX2 }\end{array}$ \\
\hline
\end{tabular}

$0006955 \quad$ Immune response $\quad 1.37 \mathrm{E}-22$

CXCL1, IFIH1, IFITM2, IFITM3, CXCL3, OAS3, TLR3, RSAD2, OAS1, IFI44L, IL32, OAS2, NFKB2, IL15, CCL5, CXCL11, IFI35, CXCL10, MYD88, TAP1, PTX3, NFIL3, DHX58, IL1A, SECTM1, IL6, SP100, BST2, IL8, CEBPG, RELB, SAMHD1, TRIM22, AIM2, PSMB8, PSMB9, DDX58, TNFSF 10, OASL, APOL1, IL20RB, VEGFA, CD274, FAIM3, GBP3, IFI6, GBP1

0006952 Defense response

3.30E-11

CXCL1, IFIH1, NMI, CXCL3, TLR3, RSAD2, IL32, IL15, CXCL11, CCL5, CXCL10, MYD88, HMOX1, TAP1, MX1, PTX3, MX2, DHX58, IL1A, IL6, SP100, IL8, CEBPG, SAMHD1, DDX58, APOL3, APOL1, IL2ORB, IFNB1, IRF7, FAIM3

$0005125 \quad$ Cytokine activity 6.08E-08

$0042981 \quad$ Regulation of apoptosis 7.10E-08

0043067

Regulation of

programmed cell death

8.78E-08

CXCL1, SECTM1, IL6, IL8, CXCL3, IL32, IL15, CXCL11, CCL5, CXCL10, TSLP, TNFSF10, IFNB1, VEGFA, IL1A

TRAF1, IFIH1, BTC, PML, PMAIP1, MYD88, HMOX1, MX1, IL1A, TXNIP, PRKCA, ARHGEF2, IL6, KLF10, CEBPG, IFI16, STAT1, BIRC3, DDIT3, PLEKHF1, TNFRSF9, XPA, DHRS2, TNFSF10, IFNB1, VEGFA, SERPINB2, FAIM3, TNFAIP3, IFI6

TRAF1, IFIH1, BTC, PML, PMAIP1, MYD88, HMOX1, MX1, IL1A, TXNIP, PRKCA, ARHGEF2, IL6, KLF10, CEBPG, IFI16, STAT1, BIRC3, DDIT3, PLEKHF1, TNFRSF9, XPA, DHRS2, TNFSF10, IFNB1, VEGFA, SERPINB2, FAIM3, TNFAIP3, IFI6

$0010941 \quad$ Regulation of cell death 9.50E-08 TRAF1, IFIH1, BTC, PML, PMAIP1, MYD88, HMOX1, MX1, IL1A, TXNIP, PRKCA, ARHGEF2, IL6, KLF10, CEBPG, IFI16, STAT1, BIRC3, DDIT3, PLEKHF1, TNFRSF9, XPA, DHRS2, TNFSF10, IFNB1, VEGFA, SERPINB2, FAIM3, TNFAIP3, IFI6

$0005615 \quad$ Extracellular space

$1.73 \mathrm{E}-07$

CXCL1, SECTM1, IL6, IL8, CXCL3, BTC, IL32, IL15, VGF, CXCL11, CCL5, CXCL10, TSLP, VEGFC, FGG, TNFSF10, APOL1, ISG15, IFNB1, HMOX1, VEGFA, SERPINB2, ANGPTL1, IL1A

$0008285 \quad$ Negative regulation of cell proliferation

2.93E-07 PRKCA, CXCL1, IL6, IL8, IFITM1, KLF10, PML, IL15, WARS, DHRS2, TNFRSF9, CTH, IL2ORB, IFNB1, HMOX1, CD274, EIF2AK2, IL1A, KLF4

$0042127 \quad$ Regulation of 5.80E-07 cell proliferation CXCL1, IFITM1, BTC, PML, IL15, CXCL10, WARS, MYD88, HMOX1, IL1A, TXNIP, PRKCA, ARHGEF2, IL6, IL8, KLF10, STAT1, TNFRSF9, DHRS2, VEGFC, CTH, ATF3, IL2ORB, IFNB1, CD274, VEGFA, EIF2AK2, KLF4

$\begin{array}{lll}0006954 & \text { Inflammatory response } & 1.65 \mathrm{E}-06 \\ 0009611 & \text { Response to wounding } & \text { 4.87E-06 } \\ 0006917 & \text { Induction of apoptosis } & 2.85 \mathrm{E}-05 \\ 0012502 & \begin{array}{l}\text { Induction of } \\ \text { programmed cell death }\end{array} & 2.95 \mathrm{E}-05 \\ & & \end{array}$

CXCL1, IL6, NMI, IL8, CXCL3, TLR3, IL15, CXCL11, CCL5, CXCL10, APOL3, MYD88, IL20RB, HMOX1, IRF7, PTX3, IL1A CXCL1, IL6, NMI, CYP1A1, IL8, CXCL3, TLR3, IL15, CXCL11, CCL5, CXCL10, APOL3, PLSCR1, FGG, MYD88, IL2ORB, HMOX1, IRF7, SERPINB2, PTX3, IL1A

PRKCA, ARHGEF2, CEBPG, KLF10, PML, IFI16, PMAIP1, STAT1, PLEKHF1, XPA, TNFRSF9, TNFSF10, IFNB1, HMOX1, MX1 PRKCA, ARHGEF2, CEBPG, KLF10, PML, IFI16, PMAIP1, STAT1, PLEKHF1, XPA, TNFRSF9, TNFSF10, IFNB1, HMOX1, MX1

False discovery rate<0.05. DEGs, differentially expressed genes; CYR61, cysteine-rich 61; GO, gene ontology.

GO and KEGG subpathway analysis of module-associated $D E G s$. To gain further insight into the function of proteins in the network module, module-associated DEGs were annotated by DAVID, and the results were presented in Table III.
The functions of DEGs in the above module were primarily associated with response to viruses (GO:0009615), immune response (GO:0006955), defense response (GO:0006952) and RNA binding (GO:0003723; listed in descending order). 
Table II. KEGG pathway enrichment analysis of upregulated DEGs in gene expression profiles of glioma cell expressing CYR61.

\begin{tabular}{|c|c|c|c|}
\hline ID & Function & P-value & DEG \\
\hline Hsa04060 & $\begin{array}{l}\text { Cytokine-cytokine } \\
\text { receptor interaction }\end{array}$ & $5.81 \mathrm{E}-07$ & $\begin{array}{l}\text { CXCL1, IL6, IL8, CXCL3, IL15, CXCL11, CCL5, } \\
\text { CXCL10, VEGFC, TNFRSF9, TSLP, TNFSF10, } \\
\text { IL2ORB, INHBE, IFNB1, VEGFA, IL1A }\end{array}$ \\
\hline Hsa04623 & $\begin{array}{l}\text { Cytosolic DNA- } \\
\text { sensing pathway }\end{array}$ & 8.67E-07 & $\begin{array}{l}\text { DDX58, IL6, IFNB1, IRF7, TREX1, CCL5, AIM2, } \\
C X C L 10, A D A R\end{array}$ \\
\hline Hsa04622 & $\begin{array}{l}\text { RIG-I-like receptor } \\
\text { signaling pathway }\end{array}$ & $6.30 \mathrm{E}-06$ & $\begin{array}{l}\text { DDX58, IFIH1, ISG15, IL8, IFNB1, IRF7, TRIM25, } \\
\text { DHX58, CXCL10 }\end{array}$ \\
\hline Hsa04620 & $\begin{array}{l}\text { Toll-like receptor } \\
\text { signaling pathway }\end{array}$ & $1.14 \mathrm{E}-05$ & $\begin{array}{l}\text { IL6, MYD88, IL8, IFNB1, IRF7, TLR3, CXCL11, } \\
\text { STAT1, CCL5, CXCL10 }\end{array}$ \\
\hline
\end{tabular}

False discovery rate<0.05. DEGs, differentially expressed genes; CYR61, cysteine-rich 61; GO, gene ontology; KEGG, Kyoto Encyclopedia of Genes and Genomes.

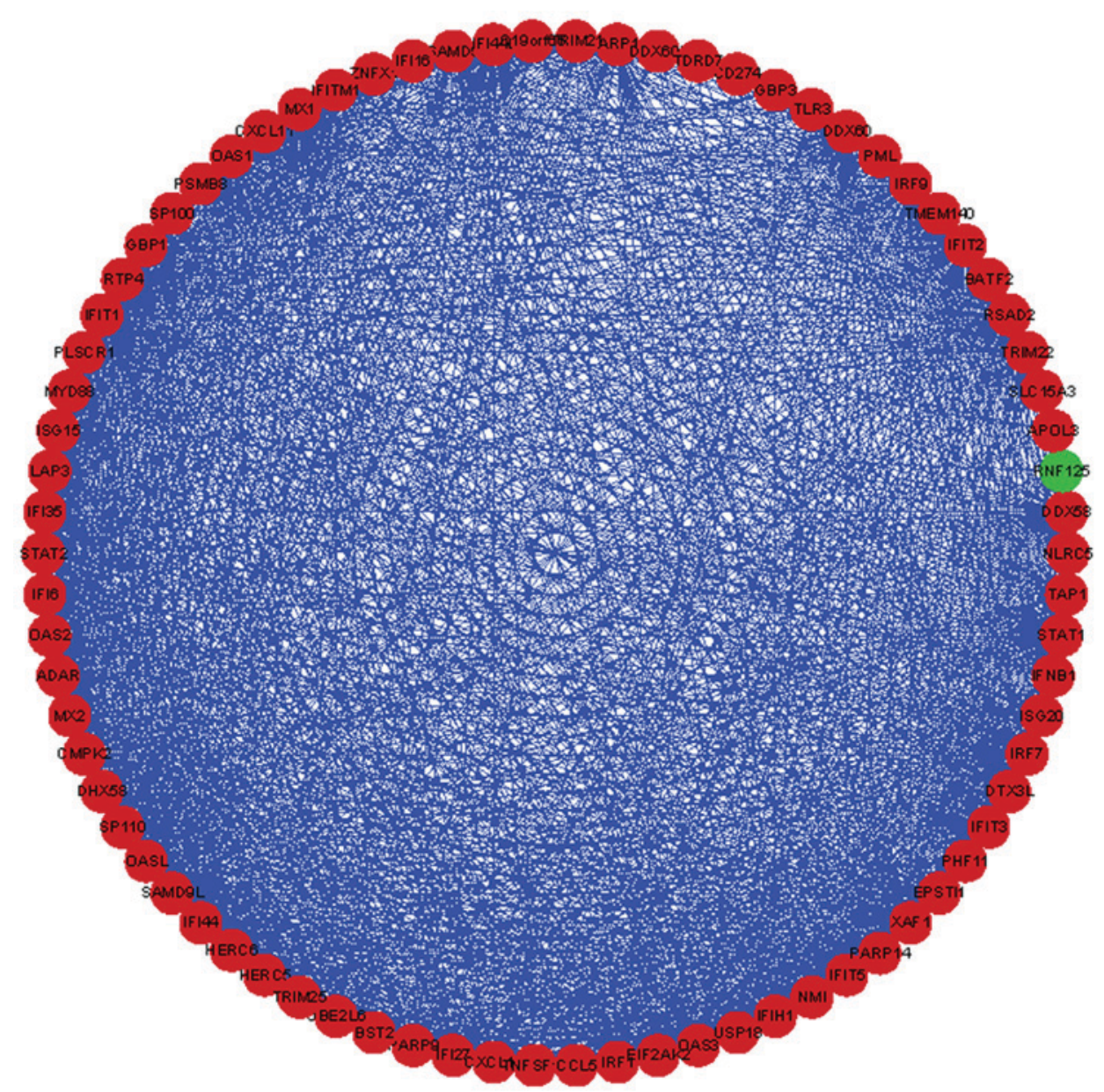

Figure 1. Module analysis based on the protein-protein interaction network for the interaction of protein molecules in glioma cells expressing cysteine-rich 61. Circle nodes represent proteins; red nodes represent upregulated DEGs; the green node represents downregulated DEGs; edges represent interactions between proteins. DEGs, differentially expressed genes.

IFIH1, IFI44 and IRF7 were connected with more than one GO term. DDX58, involved in all enriched GO terms, was involved in the innate immune defense against viruses.

The KEGG subpathway enrichment analysis of module-associated DEGs revealed three subpathways (Table IV): RIG-I-like receptor (11.5\%, hsa04622), Toll-like receptor (10.3\%, hsa04620) and cytosolic DNA-sensing (7.7\%, hsa04623). IFNBI, IRF7 and DDX58 were additionally involved in these subpathways.

Detection of TFs of DEGs. A total of seven TFs were identified by analyzing the upstream regulatory elements of the upregulated 
Table III. GO term enrichment analysis based on module-associated DEGs.

\begin{tabular}{|c|c|c|c|}
\hline GO ID & Function & P-value & DEG \\
\hline 0009615 & Response to virus & $6.77 \mathrm{E}-28$ & $\begin{array}{l}\text { IFIH1, BST2, TLR3, RSAD2, IFI44, IFI16, CCL5, } \\
\text { STAT1, TRIM22, IFI35, ISG20, STAT2, DDX58, } \\
\text { IRF9, PLSCR1, ISG15,IFNB1, IRF7, MX1, } \\
\text { EIF 2AK2, MX2 }\end{array}$ \\
\hline 0006955 & Immune response & $2.85 \mathrm{E}-17$ & $\begin{array}{l}\text { IFIH1, OAS3, RSAD2, TLR3, IFI44L, OAS1, } \\
\text { OAS2, CXCL11, CCL5, IFI35, CXCL10, RNF 125, } \\
\text { MYD88, TAP1, DHX58, SP100, BST2, TRIM22, } \\
\text { PSMB8, DDX58, OASL, TNFSF 10, CD274, GBP3, } \\
\text { IFI6, GBP1 }\end{array}$ \\
\hline 0006952 & Defense response & $6.35 \mathrm{E}-09$ & $\begin{array}{l}\text { IFIH1, NMI, SP100, RSAD2, TLR3, CXCL11, } \\
\text { CCL5, CXCL10, DDX58, APOL3, MYD88, } \\
\text { IFNB1, IRF7, TAP1, MX1, MX2, DHX58 }\end{array}$ \\
\hline 0003723 & RNA binding & $1.54 \mathrm{E}-05$ & $\begin{array}{l}\text { IFIH1, OAS3, TLR3, OAS1, OAS2, TRIM21, } \\
\text { ISG20,DDX58, OASL, DDX60, DDX60L, } \\
\text { EIF2AK2, DHX58, ADAR }\end{array}$ \\
\hline
\end{tabular}

DEGs, differentially expressed genes; GO, gene ontology.

Table IV. KEGG subpathway enrichment analysis based on module-associated DEGs.

\begin{tabular}{llll}
\hline ID & Signaling pathway & P-value & \multicolumn{1}{c}{ DEG } \\
\hline Hsa04622 & RIG-I-like receptor & $1.74 \mathrm{E}-09$ & RNF125, DDX58, IFIH1, ISG15, IFNB1, IRF7, \\
Hsa04620 & Toll-like receptor & TRIM25, DHX58, CXCL10 \\
Hsa04623 & Cytosolic DNA-sensing & MYD88, IFNB1, IRF7, TLR3, CXCL11, CCL5, \\
& & & STAT1, CXCL10 \\
\end{tabular}

DEGs, differentially expressed genes; KEGG, Kyoto Encyclopedia of Genes and Genomes.

DEGs: interferon consensus sequence-binding protein, IFN-stimulated gene factor-3 (ISGF-3), hypoxia inducible factor $(H I F) 1$, Endothelial PAS Domain Protein 1 (EPAS1), $M Y C$, aryl hydrocarbon receptor nuclear translocator (ARNT) and $M Y C$-associated factor X $(M A X)$. ISGF-3 is composed of STAT1, STAT2, and $p 48-I S G F 3 \gamma(19,20)$. The differentially expressed levels of the DEGs encoding TFs in glioma cells between the Dox and Nodox groups are presented in Fig. 2. In glioma cells expressing CYR61, IRF2 and ARNT were downregulated, whereas the others were upregulated.STAT1, STAT2 and $M Y C$ were significantly upregulated in cells expressing CYR61 cells compared with Nodox cells. However, no TFs of the downregulated DEGs were identified within the selected parameters threshold.

\section{Discussion}

Comparison of gene expression profiles between human glioma cells expressing CYR61 and those not expressing CYR61 demonstrated there were more upregulated DEGs (230/258) than downregulated DEGs (28/258). GO functional and KEGG pathway enrichment analysis revealed that the majority of upregulated DEGs (including IFNB1, IFI44, IFI16, IRF7) were associated with immune, defense and virus responses, and with cytokine-cytokine receptor, cytosolic DNA-sensing, RIG-I-like receptor and Toll-like receptor signaling pathways. However, none of the downregulated DEGs were enriched in any GO terms or KEGG subpathways. Module analysis of the PPI network revealed that STAT1 and DDX58 had high connection degrees in the PPI network. Additionally, a total of seven TFs (ICSBP, ISGF-3, HIF1, EPAS1, MYC, ARNT and $M A X)$ were identified by detecting TF binding sites within the upregulated DEGs.

GO term and KEGG subpathway enrichment analyses revealed that CYR61 expression in human glioma cells was associated with upregulation of DEGs encoding type I IFNs and IFN-induced proteins. These overexpressed genes included IFNBI, genes encoding IFN-induced proteins (IFII6, IFI27, IFI44 and IFITMI) and IRFs (IRF1, IRF7 and IRF9). Haseley et al (12) previously demonstrated that CYR61 expression results in the induction of type I IFN responsive genes. $I F N B 1$, a type I IFN, is primarily known for its antiviral activity, and additionally regulates a number of anti-inflammatory and immunomodulatory functions (21). Viral infection may 


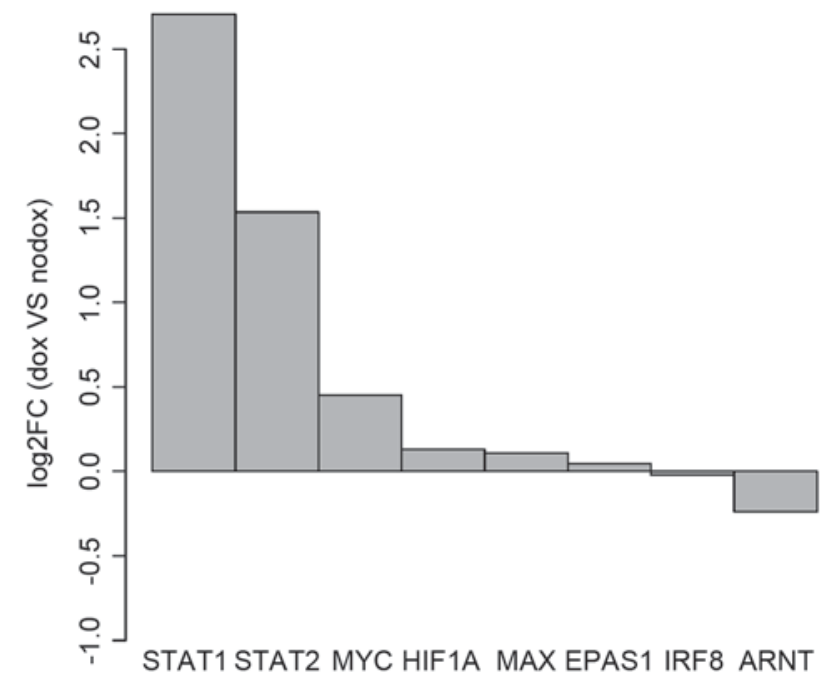

Figure 2. Expression differences in LN229 human glioma cells. Log2FC of upregulated differentially expressed genes in cells expressing CYR61 (Dox) and those not expressing CYR61 (Nodox). FC, fold change; Dox, doxycycline; Nodox, no doxycycline; STAT, signal transducer and activator of transcription; HIF1A, hypoxia inducible factor 1A; EPAS1, endothelial PAS domain-containing protein 1; IRF8, interferon regulatory factor 8; ARNT, aryl hydrocarbon receptor nuclear translocator; MAX, MYC-associated factor X.

induce transcription of multiple IFN genes, a response that is partially mediated by IRFs $(22,23)$. Yoshino et al $(24)$ reported that upregulation of IRF1 may be important for susceptibility to $I F N B$, which may regulate cell growth in diffusely infiltrating astrocytomas. IRF7 is a primary regulator of type-I IFN-dependent immune responses (25). Colina et al (26) demonstrated that translational control of IRF7 is critical for the induction of type I IFN, which indirectly is associated with the expression of CYR61.

The PPI network analysis and module analysis demonstrated that proteins with high connection degrees were encoded by STAT1 and DDX58. STAT1, an important node in the protein network of the present study, is a critical factor in the Janus-kinase-STAT signaling transduction pathway for the type IIFNs (IFNA and IFNB) and type II IFN (IFNG) (27). Additionally, STAT1 is considered a tumor suppressor (28). Huang et al (29) suggested that tumor-intrinsic STAT1 is an important mediator of anti-angiogenic signals, including IFN, and STAT1 expressed by tumor cells is a negative regulator of tumor angiogenesis, growth and metastasis. DDX58 belongs to the DEAD/H box family and has diverse roles in regulating gene expression and cellular processes (30). Previous studies have reported that DDX58 is crucial in the RIG-I-like receptor signaling pathway (31) and the innate immune response (32). Thus, upregulation of DDX58 may result in induction of the innate immune function in human glioma cells. In addition, the present study demonstrated that DDX58 is involved in response to viruses, defense response and the cytosolic DNA-sensing signaling pathway. Therefore, overexpression of proteins associated with immunity in gliomas expressing CYR61 induce a stronger immune response against viruses.

In conclusion, the results of the present study indicated that IFNB1, genes encoding IFN-induced proteins (IFII6, IFI27,
IFI44 and IFITMI), IRFs (IRF1, IRF7 and IRF9), STAT1 and $D D X 58$ are associated with CYR61 expression in glioma cells. Therefore, these genes may be critical for maintaining the role of CYR61 in cancer progression, and are potential therapeutic targets for the treatment of glioma. However, further studies are required to validate these findings.

\section{References}

1. Yang GP and Lau LF: Cyr61, product of a growth factor-inducible immediate early gene, is associated with the extracellular matrix and the cell surface. Cell Growth Differ 2: 351-357, 1991.

2. Chen CC and Lau LF: Functions and mechanisms of action of CCN matricellular proteins. Int J Biochem Cell Biol 41: 771-783, 2009.

3. Xie D, Yin D, Wang HJ, Liu GT, Elashoff R, Black K and Koeffler HP: Levels of expression of CYR61 and CTGF are prognostic for tumor progression and survival of individuals with gliomas. Clin Cancer Res 10: 2072-2081, 2004.

4. Sin WC, Bechberger JF, Rushlow WJ and Naus CC: Dose-dependent differential upregulation of CCN1/Cyr61 and $\mathrm{CCN} 3 / \mathrm{NOV}$ by the gap junction protein Connexin43 in glioma cells. J Cell Biochem 103: 1772-1782, 2008.

5. Kireeva ML, Mo FE, Yang GP and Lau LF: Cyr61, a product of a growth factor-inducible immediate-early gene, promotes cell proliferation, migration, and adhesion. Mol Cell Biol 16: 1326-1334, 1996.

6. Babic AM, Kireeva ML, Kolesnikova TV and Lau LF: CYR61, a product of a growth factor-inducible immediate early gene, promotes angiogenesis and tumor growth. Proc Natl Acad Sci USA 95: 6355-6360, 1998.

7. Tsai MS, Bogart DF, Castañeda JM, Li P and Lupu R: Cyr61 promotes breast tumorigenesis and cancer progression. Oncogene 21: 8178-8185, 2002

8. Xie D, Yin D, Tong X, O'Kelly J, Mori A, Miller C, Black K, Gui D, Said JW and Koeffler HP: Cyr61 is overexpressed in gliomas and involved in integrin-linked kinase-mediated Akt and beta-catenin-TCF/Lef signaling pathways. Cancer Res 64: 1987-1996, 2004.

9. Chen Y and Du XY: Functional properties and intracellular signaling of CCN1/Cyr61. J Cell Biochem 100: 1337-1345, 2007.

10. Goodwin CR, Lal B, Zhou X, Ho S, Xia S, Taeger A, Murray J and Laterra J: Cyr61 mediates hepatocyte growth factor-dependent tumor cell growth, migration, and akt activation. Cancer Res 70: 2932-2941, 2010.

11. Jeansonne D, Pacifici M, Lassak A, Reiss K, Russo G, Zabaleta J and Peruzzi F: Differential Effects of MicroRNAs on glioblastoma growth and migration. Genes (Basel) 4: 46-64, 2013.

12. Haseley A, Boone S, Wojton J, Yu L, Yoo JY, Yu J, Kurozumi K, Glorioso JC, Caligiuri MA and Kaur B: Extracellular matrix protein CCN1 limits oncolytic efficacy in glioma. Cancer Res 72: 1353-1362, 2012

13. Gautier L, Cope L, Bolstad BM and Irizarry RA: affy-analysis of Affymetrix GeneChip data at the probe level. Bioinformatics 20 : 307-315, 2004.

14. Smyth GK: Limma: Linear models for microarray data. In: Bioinformatics and computational biology solutions using $\mathrm{R}$ and Bioconductor. Springer, pp397-420, 2005.

15. Huang da W, Sherman BT and Lempicki RA: Systematic and integrative analysis of large gene lists using DAVID bioinformatics resources. Nat Protoc 4: 44-57, 2008.

16. Franceschini A, Szklarczyk D, Frankild S, Kuhn M, Simonovic M, Roth A, Lin J, Minguez P, Bork P, von Mering C and Jensen LJ: STRING v9. 1: Protein-protein interaction networks, with increased coverage and integration. Nucleic Acids Res 41: D808-D815, 2013.

17. Saito R, Smoot ME, Ono K, Ruscheinski J, Wang PL, Lotia S, Pico AR, Bader GD and Ideker T: A travel guide to Cytoscape plugins. Nat Methods 9: 1069-1076, 2012.

18. Loots GG, Ovcharenko I, Pachter L, Dubchak I and Rubin EM: rVista for comparative sequence-based discovery of functional transcription factor binding sites. Genome Res 12: 832-839, 2002.

19. Wong LH, Krauer KG, Hatzinisiriou I, Estcourt MJ, Hersey P, Tam ND, Edmondson S, Devenish RJ and Ralph SJ: Interferon-resistant human melanoma cells are deficient in ISGF3 components, STAT1, STAT2, and p48-ISGF3gamma. J Biol Chem 272: 28779-28785, 1997. 
20. Kessler DS, Veals SA, Fu XY and Levy DE: Interferon-alpha regulates nuclear translocation and DNA-binding affinity of ISGF3, a multimeric transcriptional activator. Genes Dev 4: $1753-1765,1990$

21. Jha AN, Singh VK, Singh R, Pati SS, Patra PK, Singh L and Thangaraj K: A rare non-synonymous c. $102 \mathrm{C}>\mathrm{G}$ SNP in the IFNB1 gene might be a risk factor for cerebral malaria in Indian populations. Infect Genet Evol 14: 369-374, 2013.

22. Foy E, Li K, Wang C, Sumpter R Jr, Ikeda M, Lemon SM and Gale M Jr: Regulation of interferon regulatory factor- 3 by the hepatitis C virus serine protease. Science 300: 1145-1148, 2003.

23. Nguyen H, Hiscott J and Pitha PM: The growing family of interferon regulatory factors. Cytokine Growth Factor Rev 8 : 293-312, 1997.

24. Yoshino A, Katayama Y, Yokoyama T, Watanabe T, Ogino A, Ota T, Komine C, Fukushima T and Kusama K: Therapeutic implications of interferon regulatory factor (IRF)-1 and IRF-2 in diffusely infiltrating astrocytomas (DIA): Response to interferon (IFN)-beta in glioblastoma cells and prognostic value for DIA. J Neurooncol 74: 249-260, 2005.

25. Honda K, Yanai H, Negishi H, Asagiri M, Sato M, Mizutani T, Shimada N, Ohba Y, Takaoka A, Yoshida N and Taniguchi T: IRF-7 is the master regulator of type-I interferon-dependent immune responses. Nature 434: 772-777, 2005.
26. Colina R, Costa-Mattioli M, Dowling RJ, Jaramillo M, Tai LH, Breitbach CJ, Martineau Y, Larsson O, Rong L, Svitkin YV, et al: Translational control of the innate immune response through IRF-7. Nature 452: 323-328, 2008.

27. Schindler C, Levy DE and Decker T: JAK-STAT signaling: From interferons to cytokines. J Biol Chem 282: 20059-20063, 2007.

28. Bromberg J: Stat proteins and oncogenesis. J Clin Invest 109: $1139-1142,2002$

29. Huang S, Bucana CD, Van Arsdall M and Fidler IJ: Stat1 negatively regulates angiogenesis, tumorigenicity and metastasis of tumor cells. Oncogene 21: 2504-2512, 2002.

30. Imaizumi T, Aratani S, Nakajima T, Carlson M, Matsumiya $T$, Tanji K, Ookawa K, Yoshida H, Tsuchida S, McIntyre TM, et al: Retinoic acid-inducible gene-I is induced in endothelial cells by LPS and regulates expression of COX-2. Biochem Biophys Res Commun 292: 274-279, 2002

31. Takeuchi $\mathrm{O}$ and Akira S: MDA5/RIG-I and virus recognition. Curr Opin Immunol 20: 17-22, 2008.

32. Xu XC: Tumor-suppressive activity of retinoic acid receptor-beta in cancer. Cancer Lett 253: 14-24, 2007. 